\title{
NAK FOR EXT AND ASCENT OF MODULE STRUCTURES
}

\author{
BENJAMIN J. ANDERSON AND SEAN SATHER-WAGSTAFF \\ (Communicated by Irena Peeva)
}

To Roger A. Wiegand on the occasion of his retirement

\begin{abstract}
We investigate the interplay between properties of Ext modules and the ascent of module structures along local ring homomorphisms. Specifically, let $\varphi:(R, \mathfrak{m}, k) \rightarrow(S, \mathfrak{m} S, k)$ be a flat local ring homomorphism. We show that if $M$ is a finitely generated $R$-module such that $\operatorname{Ext}_{R}^{i}(S, M)$ satisfies NAK (e.g. if $\operatorname{Ext}_{R}^{i}(S, M)$ is finitely generated over $S$ ) for $i=1, \ldots, \operatorname{dim}_{R}(M)$, then $\operatorname{Ext}_{R}^{i}(S, M)=0$ for all $i \neq 0$ and $M$ has an $S$-module structure that is compatible with its $R$-module structure via $\varphi$. We provide explicit computations of $\operatorname{Ext}_{R}^{i}(S, M)$ to indicate how large it can be when $M$ does not have a compatible $S$-module structure.
\end{abstract}

\section{INTRODUCTION}

Throughout this paper $(R, \mathfrak{m}, k)$ and $(S, \mathfrak{n}, l)$ are commutative noetherian local rings. Given an $R$-module $M$, the $\mathfrak{m}$-adic completion of $M$ is denoted $\widehat{M}$.

The genesis for this paper begins with the following result of Buchweitz and Flenner [2, Theorem 2.3].

Theorem 1.1. Let $M$ be an $R$-module. If $M$ is $\mathfrak{m}$-adically complete, then for each flat $R$-module $F$ one has $\operatorname{Ext}_{R}^{i}(F, M)=0$ for all $i \geqslant 1$.

The converse fails with no extra assumptions on $M$, as we see next.

Example 1.2. Let $M$ be a non-zero injective $R$-module, and assume that $R$ has positive depth (e.g., $R$ is a domain and not a field). Then $\operatorname{Ext}_{R}^{i}(-, M)=0$ for all $i \geqslant 1$. However, the fact that $M$ is injective implies that it is divisible. Thus, we have $M=\mathfrak{m} M$, and it follows that $\widehat{M}=0 \neq M$, so $M$ is not complete.

The next result of Frankild and Sather-Wagstaff [7, Corollary 3.5] shows that the converse to Theorem 1.1 does hold when $M$ is finitely generated.

Theorem 1.3. Let $M$ be a finitely generated $R$-module. Then the following conditions are equivalent:

(i) $M$ is $\mathfrak{m}$-adically complete.

(ii) $\operatorname{Ext}_{R}^{i}(F, M)=0$ for all $i \geqslant 1$ for each flat $R$-module $F$.

(iii) $\operatorname{Ext}_{R}^{i}(\widehat{R}, M)=0$ for all $i \geqslant 1$.

Received by the editors November 30, 2011 and, in revised form, May 14, 2012.

2010 Mathematics Subject Classification. Primary 13B40, 13D07; Secondary 13D02.

Key words and phrases. Ascent, Ext, flat homomorphism, NAK.

This material is based on work supported by North Dakota EPSCoR and National Science Foundation Grant EPS-0814442. 
It is worth noting that the proof of this result is quite technical, relying heavily on the machinery of derived local homology and derived local cohomology.

Since the module $M$ in Theorem 1.3 is finitely generated, a standard result shows that condition (1) is equivalent to the following: $M$ has an $\widehat{R}$-module structure that is compatible with its $R$-module structure via the natural map $R \rightarrow \widehat{R}$. Thus, we consider the following ascent question, focusing on homological conditions.

Question 1.4. Given a ring homomorphism $\varphi: R \rightarrow S$, what conditions on an $R$-module $M$ guarantee that it has an $S$-module structure compatible with its $R$ module structure via $\varphi$ ?

In the setting of Question 1.4, the module $S \otimes_{R} M$ has a natural $S$-module structure. Thus, if one had an $R$-module isomorphism $M \cong S \otimes_{R} M$, then one could transfer the $S$-module structure from $S \otimes_{R} M$ to $M$. One can similarly inflict an $S$-module structure on $M$ if $M \cong \operatorname{Hom}_{R}(S, M)$. The following result of Frankild, Sather-Wagstaff, and R. Wiegand [8, Main Theorem 2.5] and Christensen and Sather-Wagstaff [4, Theorem 3.1 and Remark 3.2] shows that when $\varphi$ is a local homomorphism with properties like those of the natural map $R \rightarrow \widehat{R}$, these are in fact the only way for $M$ to admit a compatible $S$-module structure.

Theorem 1.5. Let $\varphi: R \rightarrow S$ be a flat local ring homomorphism such that the induced map $R / \mathfrak{m} \rightarrow S / \mathfrak{m} S$ is an isomorphism. Let $M$ be a finitely generated $R$-module, and consider the following conditions:

(i) $M$ has an $S$-module structure compatible with its $R$-module structure via $\varphi$.

(ii) $\operatorname{Ext}_{R}^{i}(S, M)=0$ for all $i \geqslant 1$.

(iii) $\operatorname{Ext}_{R}^{i}(S, M)$ is finitely generated over $R$ for $i=1, \ldots, \operatorname{dim}(M)$.

(iv) The natural map $\operatorname{Hom}_{R}(S, M) \rightarrow M$ given by $f \mapsto f(1)$ is an isomorphism.

(v) The natural map $M \rightarrow S \otimes_{R} M$ given by $m \mapsto 1 \otimes m$ is an isomorphism.

(vi) $S \otimes_{R} M$ is finitely generated over $R$.

(vii) $\operatorname{Ext}_{R}^{i}(S, M)$ is finitely generated over $S$ for $i=1, \ldots, \operatorname{dim}_{R}(M)$.

Then conditions (ii)-(vi) are equivalent and imply condition (vii). If $R$ is Gorenstein, then conditions (ii)-(vii) are equivalent.

The proof of this result is less technical than that of Theorem 1.3 but it does use the Amplitude Inequality of Foxby, Iyengar, and Iversen [6, 9] - a consequence of the New Intersection Theorem - and the derived Gorenstein injective dimension.

The next result is the main theorem of the current paper. It contains several improvements on Theorem 1.5. First, it removes the Gorenstein hypothesis for the implication (vii) $\Longrightarrow$ (ii). Second, it further relaxes the conditions on the Ext-modules needed to obtain an $S$-module structure on $M$. Third, the proof is significantly less technical than the proofs of these earlier results, relying only on basic properties of Koszul complexes. It is proved in Theorem 3.2

Definition 1.6. An $R$-module $N$ satisfies $N A K$ if $N=0$ or $N / \mathfrak{m} N \neq 0$.

Theorem 1.7. In Theorem 3.2 the conditions (ii)-(vii) are equivalent, and they are equivalent to the following:

(viii) $\operatorname{Ext}_{R}^{i}(S, M)$ satisfies $N A K$ for $i=1, \ldots, \operatorname{dim}_{R}(M)$. 
We conclude this introduction by outlining the contents of the paper. Section 2 summarizes foundational material needed for the proof of Theorem 3.2. Section 3 is devoted to the proof of this result. Finally, Section 4 consists of an example demonstrating how large $\operatorname{Ext}_{R}^{i}(S, M)$ is, even over a relatively small ring.

\section{BACKROUND MATERIAL}

Most of our definitions and notational conventions come from [3,5]. For the sake of clarity, we specify a few items here.

Convention 2.1. We index chain complexes of $R$-modules (" $R$-complexes" for short) homologically:

$$
Y=\cdots \stackrel{\partial_{i+1}^{Y}}{\longrightarrow} Y_{i} \stackrel{\partial_{i}^{Y}}{\longrightarrow} Y_{i-1} \stackrel{\partial_{i-1}^{Y}}{\longrightarrow} \cdots .
$$

Given an $R$-complex $Y$ and an integer $n$, the nth suspension (or shift) of $Y$ is denoted $\Sigma^{n} Y$ and has $\left(\Sigma^{n} Y\right)_{i}:=Y_{i-n}$. We set $\Sigma Y:=\Sigma^{1} Y$.

For two $R$-complexes $Y$ and $Z$, our indexing protocol extends to the Homcomplex $\operatorname{Hom}_{R}(Y, Z)$, so that we have $\operatorname{Hom}_{R}(Y, Z)_{i}=\prod_{j \in \mathbb{Z}} \operatorname{Hom}_{R}\left(Y_{j}, Z_{j+i}\right)$ for each $i \in \mathbb{Z}$. As usual, the tensor product $Y \otimes_{R} Z$ has $\left(Y \otimes_{R} Z\right)_{i}=\prod_{j \in \mathbb{Z}} Y_{j} \otimes_{R} Z_{i-j}$.

Given an element $x \in R$, the Koszul complex $K^{R}(x)$ is $0 \rightarrow R \stackrel{x}{\rightarrow} R \rightarrow 0$, concentrated in degrees 0 and 1 . If $\mathbf{x}=x_{1}, \ldots, x_{n}$ is a sequence in $R$, then the Koszul complex $K^{R}(\mathbf{x})$ is defined inductively: $K^{R}(\mathbf{x})=K^{R}\left(x_{1}, \ldots, x_{n-1}\right) \otimes_{R} K^{R}\left(x_{n}\right)$.

For each morphism of $R$-complexes (i.e., a chain map) $f: Y \rightarrow Z$, the mapping cone Cone $(f)$ gives rise to a short exact sequence $0 \rightarrow Z \rightarrow \operatorname{Cone}(f) \rightarrow \Sigma Y \rightarrow 0$, and hence an associated long exact sequence on homology. Given an element $x \in R$, the mapping cone associated to the morphism $Y \stackrel{x}{\rightarrow} Y$ is isomorphic to $K^{R}(x) \otimes_{R} Y$. A morphism $f: Y \rightarrow Z$ is a quasiisomorphism if the induced map $\mathrm{H}_{i}(f): \mathrm{H}_{i}(Y) \rightarrow$ $\mathrm{H}_{i}(Z)$ on homology is an isomorphism for each $i \in \mathbb{Z}$.

Lemma 2.2. Let $i_{0}$ be a fixed integer, let $\boldsymbol{x}=x_{1}, \ldots, x_{n}$ be a sequence in $R$, and let $Y$ be an $R$-complex such that $\mathrm{H}_{i}(Y)=0$ for all $i<i_{0}$. Then $\mathrm{H}_{i}\left(K^{R}(\boldsymbol{x}) \otimes_{R} Y\right)=0$ for all $i<i_{0}$ and $\mathrm{H}_{i_{0}}\left(K^{R}(\boldsymbol{x}) \otimes_{R} Y\right) \cong \mathrm{H}_{i_{0}}(Y) /(\boldsymbol{x}) \mathrm{H}_{i_{0}}(Y)$.

Proof. Argue by induction on $n$, using the long exact sequence in homology associated to the short exact sequence $0 \rightarrow Y \rightarrow K^{R}\left(x_{1}\right) \otimes_{R} Y \rightarrow \Sigma Y \rightarrow 0$.

Lemma 2.3. Let $\varphi: R \rightarrow S$ be a flat local ring homomorphism such that the induced map $R / \mathfrak{m} \rightarrow S / \mathfrak{m} S$ is an isomorphism. Let $\mathbf{x}=x_{1}, \ldots, x_{n} \in R$ be a generating sequence for $\mathfrak{m}$.

(a) The natural map $K^{R}(\boldsymbol{x}) \rightarrow S \otimes_{R} K^{R}(\boldsymbol{x})$ is a quasiisomorphism.

(b) For each bounded above complex $J$ of injective $R$-modules, the induced map $\operatorname{Hom}_{R}\left(S \otimes_{R} K^{R}(\boldsymbol{x}), J\right) \rightarrow \operatorname{Hom}_{R}\left(K^{R}(\boldsymbol{x}), J\right)$ is a quasiisomorphism.

Proof. Part (国) is from [8, 2.3], and part (b) follows from this by a standard property of bounded above complexes of injective $R$-modules.

The next result is from [8, proof of Theorem 2.5].

Lemma 2.4. Let $\varphi: R \rightarrow S$ be a flat ring homomorphism, and let $M$ be an $R$ module. Then $\operatorname{Ext}_{R}^{i}(S, M)=0$ for all $i>\operatorname{dim}\left(R / \operatorname{Ann}_{R}(M)\right)$. In particular, if $M$ is finitely generated, then $\operatorname{Ext}_{R}^{i}(S, M)=0$ for all $i>\operatorname{dim}_{R}(M)$. 


\section{Proof of Theorem 1.7}

Let $\varphi: R \rightarrow S$ be a local ring homomorphism. The term "satisfies NAK" is defined in Definition [1.6. Given an $S$-module $N$, if $N$ satisfies NAK as an $S$ module, then it satisfies NAK as an $R$-module because of the epimorphism $N / \mathfrak{m} N=$ $N / \mathfrak{m} S N \rightarrow N / \mathfrak{n} N$. Furthermore, this reasoning shows that the converse holds if $\mathfrak{m} S=\mathfrak{n}$, e.g., if the induced map $R / \mathfrak{m} \rightarrow S / \mathfrak{m} S$ is an isomorphism.

Lemma 3.1. Let $\varphi: R \rightarrow S$ be a flat local ring homomorphism such that the induced map $R / \mathfrak{m} \rightarrow S / \mathfrak{m} S$ is an isomorphism. Let $M$ be an $R$-module, and let $z \geqslant 1$. If $\operatorname{Ext}_{R}^{i}(S, M)=0$ for all $i>z$ and $\operatorname{Ext}_{R}^{z}(S, M)$ satisfies $N A K$, then $\operatorname{Ext}_{R}^{z}(S, M)=0$.

Proof. Let $\mathbf{x}=x_{1}, \ldots, x_{n} \in R$ be a generating sequence for $\mathfrak{m}$, and let $J$ be an $R$-injective resolution of $M$. By assumption we have $\mathrm{H}_{-i}\left(\operatorname{Hom}_{R}(S, J)\right) \cong$ $\operatorname{Ext}_{R}^{i}(S, M)=0$ for all $i>z$, so we have $\mathrm{H}_{-i}\left(K^{R}(\mathbf{x}) \otimes_{R} \operatorname{Hom}_{R}(S, J)\right)=0$ for all $i>z$ and

$$
\begin{aligned}
\mathrm{H}_{-z}\left(K^{R}(\mathbf{x}) \otimes_{R} \operatorname{Hom}_{R}(S, J)\right) & \cong \mathrm{H}_{-z}\left(\operatorname{Hom}_{R}(S, J)\right) / \mathbf{x H}_{-z}\left(\operatorname{Hom}_{R}(S, J)\right) \\
& \cong \operatorname{Ext}_{R}^{z}(S, M) / \mathbf{x E x t}_{R}^{z}(S, M)
\end{aligned}
$$

by Lemma 2.2 .

We claim that $\operatorname{Ext}_{R}^{z}(S, M) / \mathrm{x}_{\operatorname{Ext}}^{z}(S, M)=0$. To see this we first notice that

$$
\begin{aligned}
K^{R}(\mathbf{x}) \otimes_{R} \operatorname{Hom}_{R}(S, J) & \cong \operatorname{Hom}_{R}\left(\operatorname{Hom}_{R}\left(K^{R}(\mathbf{x}), R\right), \operatorname{Hom}_{R}(S, J)\right) \\
& \cong \operatorname{Hom}_{R}\left(\Sigma^{-n} K^{R}(\mathbf{x}), \operatorname{Hom}_{R}(S, J)\right) \\
& \cong \Sigma^{n} \operatorname{Hom}_{R}\left(K^{R}(\mathbf{x}), \operatorname{Hom}_{R}(S, J)\right) \\
& \cong \Sigma^{n} \operatorname{Hom}_{R}\left(S \otimes_{R} K^{R}(\mathbf{x}), J\right) \\
& \simeq \Sigma^{n} \operatorname{Hom}_{R}\left(K^{R}(\mathbf{x}), J\right) \\
& \cong \operatorname{Hom}_{R}\left(\Sigma^{-n} K^{R}(\mathbf{x}), J\right) \\
& \cong \operatorname{Hom}_{R}\left(\operatorname{Hom}_{R}\left(K^{R}(\mathbf{x}), R\right), J\right) \\
& \cong K^{R}(\mathbf{x}) \otimes_{R} J
\end{aligned}
$$

The first and last isomorphisms are by Hom-evaluation; see, e.g., 11, B.2. Lemma]. The fourth isomorphism is by Hom-tensor evaluation, and the other isomorphisms follow from properties of the Koszul complex. The quasiisomorphism in the fifth step is from Lemma $2.3($ b) .

This sequence explains the second isomorphism in the next display:

$$
\begin{aligned}
\operatorname{Ext}_{R}^{z}(S, M) / \operatorname{xExt}_{R}^{z}(S, M) & \cong \mathrm{H}_{-z}\left(K^{R}(\mathbf{x}) \otimes_{R} \operatorname{Hom}_{R}(S, J)\right) \\
& \cong \mathrm{H}_{-z}\left(K^{R}(\mathbf{x}) \otimes_{R} J\right) \\
& \cong \mathrm{H}_{-z}\left(K^{R}(\mathbf{x}) \otimes_{R} M\right) \\
& =0 .
\end{aligned}
$$

The first isomorphism comes from the first paragraph of this proof. The second isomorphism follows from the fact that the quasiisomorphism $M \stackrel{\simeq}{\longrightarrow} J$ is respected by $K^{R}(\mathbf{x}) \otimes_{R}-$, and the vanishing is due to the assumption $z \geqslant 1$.

As $\operatorname{Ext}_{R}^{z}(S, M)$ satisfies NAK, the claim implies $\operatorname{Ext}_{R}^{z}(S, M)=0$, as desired. 
Here is Theorem 1.7 from the introduction.

Theorem 3.2. Let $\varphi: R \rightarrow S$ be a flat local ring homomorphism such that the induced map $R / \mathfrak{m} \rightarrow S / \mathfrak{m} S$ is an isomorphism, and let $M$ be a finitely generated $R$ module. If $\operatorname{Ext}_{R}^{i}(S, M)$ satisfies $N A K$ for $i=1, \ldots, \operatorname{dim}_{R}(M)$, then $\operatorname{Ext}_{R}^{i}(S, M)=$ 0 for all $i \neq 0$ and $M$ has an $S$-module structure compatible with its $R$-module structure via $\varphi$.

Proof. Set $z=\sup \left\{i \geqslant 0 \mid \operatorname{Ext}_{R}^{i}(S, M) \neq 0\right\} 1$ Lemma 2.4 implies that $z \leqslant$ $\operatorname{dim}_{R}(M)$. Note that $z \leqslant 0$; if $z \geqslant 1$, then Lemma 3.1 implies that $\operatorname{Ext}_{R}^{z}(S, M)=0$, a contradiction. It follows that $\operatorname{Ext}_{R}^{i}(S, M)=0$ for all $i \neq 0$, and the remaining conclusions follow from Theorem 1.5 .

Remark 3.3. As we note in the introduction, our proof of this theorem removes the need to invoke the Amplitude Inequality in the proof of Theorem 1.5. Indeed, the Amplitude Inequality is used in the implication (iii) $\Longrightarrow$ (iii), which we prove directly in the proof of Theorem 3.2 .

Remark 3.4. One can paraphrase Theorem 3.2 as follows: In Theorem 1.5(iii) one can replace the phrase "is finitely generated over $R$ " with the phrase "satisfies NAK". It is natural to ask whether the same replacement can be done in Theorem 1.5(vi). In fact, this cannot be done because, given a finitely generated $R$ module $M$, the $S$-module $S \otimes_{R} M$ is finitely generated, so it automatically satisfies NAK, regardless of whether $M$ has a compatible $S$-module structure.

\section{ExPlicit COMPUTATIONS}

Given a ring homomomorphism $\varphi: R \rightarrow S$ as in Theorem 3.2 , if $M$ is a finitely generated $R$-module that does not have a compatible $S$-module structure, then we know that $\operatorname{Ext}_{R}^{i}(S, M)$ does not satisfy NAK for some $i$. Hence, this Ext-module is quite large. This section is devoted to a computation showing how large this Extmodule is when $R \neq \widehat{R}=S$, even for the simplest ring $R$, e.g., for $R=k[X]_{(X)}$, where $k$ is a field or for $\mathbb{Z}_{p \mathbb{Z}}$. See Remark 4.3.

Lemma 4.1. Let $\varphi: R \rightarrow S$ be a faithfully flat ring homomorphism, and let $C$ be an $R$-module. Let $\mathfrak{m}$ be a maximal ideal of $R$, and assume that $C$ is $\mathfrak{m}$-adically complete.

(a) Then $\operatorname{Ext}_{R}^{i}(S / R, C)=0=\operatorname{Ext}_{R}^{i}(S, C)$ for all $i \geqslant 1$.

(b) If $R$ is local and the natural map $R / \mathfrak{m} \rightarrow S / \mathfrak{m} S$ is an isomorphism, then $\operatorname{Hom}_{R}(S / R, C)=0$, the module $C$ has an $S$-module structure compatible with its $R$-module structure via $\varphi$, and the natural maps $C \rightarrow \operatorname{Hom}_{R}(S, C) \rightarrow C$ are inverse isomorphisms.

(c) If $R$ is local, then $\operatorname{Ext}_{R}^{i}(\widehat{R} / R, \widehat{R})=0=\operatorname{Ext}_{R}^{i+1}(\widehat{R}, \widehat{R})$ for all $i \geqslant 0$, and the natural maps $\widehat{R} \rightarrow \operatorname{Hom}_{R}(\widehat{R}, \widehat{R}) \rightarrow \widehat{R}$ are inverse isomorphisms.

Proof. (a) The fact that $S$ is faithfully flat over $R$ implies that $\varphi$ is a pure monomorphism, and it follows that $S / R$ is flat over $R$; see [10, Theorem 7.5]. Since $S$ and $S / R$ are flat over $R$, the desired vanishing follows from Theorem 1.1

(b) Assume that $R$ is local and the natural map $R / \mathfrak{m} \rightarrow S / \mathfrak{m} S$ is an isomorphism. In particular, the ideal $\mathfrak{m} S \subset S$ is maximal.

\footnotetext{
${ }^{1}$ We take the supremum here instead of the maximum since we do not know a priori whether the set $\left\{i \geqslant 0 \mid \operatorname{Ext}_{R}^{i}(S, M) \neq 0\right\}$ is non-empty.
} 
Claim: The natural map $\widehat{\varphi}: \widehat{R} \rightarrow \widehat{S}$ between $\mathfrak{m}$-adic completions is an isomorphism. To see this, first note that the fact that $\mathfrak{m} S$ is maximal implies that $\widehat{S}$ is local with maximal ideal $\mathfrak{m} \widehat{S}$. Furthermore, the induced map $\widehat{R} / \mathfrak{m} \widehat{R} \rightarrow \widehat{S} / \mathfrak{m} \widehat{S}$ is equivalent to the isomorphism $R / \mathfrak{m} \rightarrow S / \mathfrak{m} S$, so it is an isomorphism. It follows from a version of Nakayama's Lemma [10, Theorem 8.4] that $\widehat{S}$ is a cyclic $\widehat{R}$-module. Since it is also faithfully flat, we deduce that $\widehat{\varphi}$ is an isomorphism, as claimed.

For each $n \in \mathbb{N}$, the induced map $R / \mathfrak{m}^{n} \rightarrow S / \mathfrak{m}^{n} S$ is an isomorphism. Indeed, this map is equivalent to the induced map $\widehat{R} / \mathfrak{m}^{n} \rightarrow \widehat{S} / \mathfrak{m}^{n} \widehat{S}$, which is an isomorphism because $\widehat{R} \cong \widehat{S}$. This justifies the last step in the following display:

$$
(S / R) \otimes_{R}\left(R / \mathfrak{m}^{n}\right) \cong\left(S \otimes_{R}\left(R / \mathfrak{m}^{n}\right)\right) /\left(R \otimes_{R}\left(R / \mathfrak{m}^{n}\right)\right) \cong\left(S / \mathfrak{m}^{n} S\right) /\left(R / \mathfrak{m}^{n}\right)=0 .
$$

This display explains the fifth isomorphism in the next sequence:

$$
\begin{aligned}
\operatorname{Hom}_{R}(S / R, C) & \cong \operatorname{Hom}_{R}\left(S / R, \lim _{n \leftarrow} C / \mathfrak{m}^{n} C\right) \\
& \cong \lim _{n \leftarrow} \operatorname{Hom}_{R}\left(S / R, C / \mathfrak{m}^{n} C\right) \\
& \cong \lim _{n \leftarrow} \operatorname{Hom}_{R}\left(S / R, \operatorname{Hom}_{R / \mathfrak{m}^{n}}\left(R / \mathfrak{m}^{n}, C / \mathfrak{m}^{n} C\right)\right) \\
& \cong \lim _{n \leftarrow} \operatorname{Hom}_{R / \mathfrak{m}^{n}}\left(\left(R / \mathfrak{m}^{n}\right) \otimes_{R}(S / R), C / \mathfrak{m}^{n} C\right) \\
& \cong \lim _{n \leftarrow} \operatorname{Hom}_{R / \mathfrak{m}^{n}}\left(0, C / \mathfrak{m}^{n} C\right) \\
& =0 .
\end{aligned}
$$

Now consider the exact sequence $0 \rightarrow R \rightarrow S \rightarrow S / R \rightarrow 0$ and part of the long exact sequence in $\operatorname{Ext}_{R}(-, C)$ :

$$
0 \rightarrow \underbrace{\operatorname{Hom}_{R}(S / R, C)}_{=0} \rightarrow \operatorname{Hom}_{R}(S, C) \rightarrow \underbrace{\operatorname{Hom}_{R}(R, C)}_{\cong C} \rightarrow \underbrace{\operatorname{Ext}_{R}^{1}(S / R, C)}_{=0} .
$$

It follows that the induced map $\alpha: \operatorname{Hom}_{R}(S, C) \rightarrow C$ is an isomorphism. It is straightforward to show that this is the evaluation map $f \mapsto f(1)$. Since $C$ is complete, the isomorphism $\widehat{R} \cong \widehat{S}$ implies that $C$ has an $S$-module structure that is compatible with its $R$-module structure via $\varphi$. From this, it follows that the map $\beta: C \rightarrow \operatorname{Hom}_{R}(S, C)$ given by $c \mapsto(s \mapsto s c)$ is a well-defined $S$-module homomorphism. Since the composition $\alpha \beta$ is the identity on $C$, it follows that $\alpha$ and $\beta$ are inverse isomorphisms.

(c) This follows from parts (国) and (b) using $C=\widehat{R}$.

Proposition 4.2. Assume that $R$ is a discrete valuation ring that is not complete, with $\mathfrak{m}=X R$. Set $E=E_{R}(k)=E_{\widehat{R}}(k)$, and consider the quotient fields $K=Q(R)$ and $\widetilde{K}=Q(\widehat{R})$. If $[\widetilde{K}: K]=\infty$, then there are an uncountable cardinal $C$ and $\widehat{R}$-module isomorphisms

$$
\operatorname{Ext}_{R}^{i}(\widehat{R}, R) \cong \begin{cases}0 & \text { if } i \neq 1, \\ E \oplus \widetilde{K}^{(C)} & \text { if } i=1,\end{cases}
$$

where $\widetilde{K}^{(C)}$ is the direct sum of copies of $\widetilde{K}$ indexed by $C$.

Proof. As a $K$-vector space and as an $R$-module, we have $\widetilde{K} \cong K^{(A)}$ for some infinite cardinal $A$. Note that since $R$ and $\widehat{R}$ are discrete valuation rings with uniformizing parameter $X$, we have $K \cong R_{X}$ and $\widetilde{K} \cong \widehat{R}_{X} \cong K \otimes_{R} \widehat{R}$. Since $K$ has no $X$-torsion, we have $\operatorname{Hom}_{R}(k, K)=0$. 
Claim 1: We have $\operatorname{Ext}_{R}^{i}(\widehat{R}, R)=0$ for all $i \neq 1$. Since $\operatorname{id}_{R}(R)=1$, it suffices to show that $\operatorname{Hom}_{R}(\widehat{R}, R)=0$. From [8, Corollary 1.7] we know that $\operatorname{Hom}_{R}(\widehat{R}, R)$ is isomorphic to a complete submodule $I \subseteq R$. Since $R$ is a discrete valuation ring, its non-zero submodules are all isomorphic to $R$, which is not complete. So we must have $\operatorname{Hom}_{R}(\widehat{R}, R) \cong I=0$.

Claim 2: There is an $R$-module isomorphism $\widehat{R} / R \cong \widetilde{K} / K$. In the following commutative diagram, the top row is a minimal $R$-injective resolution of $R$, and the bottom row is a minimal $\widehat{R}$-injective resolution of $\widehat{R}$ :

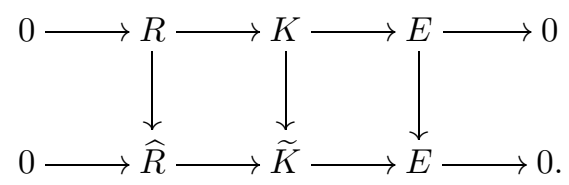

The Snake Lemma yields an $R$-module isomorphism $\widehat{R} / R \cong \widetilde{K} / K$.

Claim 3: We have an $R$-module isomorphism $\operatorname{Hom}_{R}(\widehat{R}, \widehat{R} / R) \cong(\widetilde{K} / K)^{(B)}$ for some infinite cardinal $B>A$. This is from the next sequence of $R$-module isomorphisms, where the first step is from Claim 2:

$$
\begin{aligned}
\operatorname{Hom}_{R}(\widehat{R}, \widehat{R} / R) & \cong \operatorname{Hom}_{R}(\widehat{R}, \widetilde{K} / K) \\
& \cong \operatorname{Hom}_{R}\left(\widehat{R}, \operatorname{Hom}_{K}(K, \widetilde{K} / K)\right) \\
& \cong \operatorname{Hom}_{K}\left(K \otimes_{R} \widehat{R}, \widetilde{K} / K\right) \\
& \cong \operatorname{Hom}_{K}(\widetilde{K}, \widetilde{K} / K) \\
& \cong \operatorname{Hom}_{K}\left(K^{(A)}, \widetilde{K} / K\right) \\
& \cong \operatorname{Hom}_{K}(K, \widetilde{K} / K)^{A} \\
& \cong(\widetilde{K} / K)^{A} \\
& \cong(\widetilde{K} / K)^{(B)}
\end{aligned}
$$

The third step is by Hom-tensor adjointness, the fourth step is from the isomorphism $\widetilde{K} \cong K \otimes_{R} \widehat{R}$ noted at the beginning of the proof, and the remaining steps are standard. Since $A$ is infinite, we must have $B>A$, as claimed.

Claim 4: There is a cardinal $C$ and an $\widehat{R}$-module isomorphism $\operatorname{Ext}_{R}^{1}(\widehat{R}, R) \cong$ $E \oplus \widetilde{K}^{(C)}$. We compute $\operatorname{Ext}_{R}^{1}(\widehat{R}, R)$ using the injective resolution of $R$ from the top row of (4.2.1). From Claim 1, this yields an exact sequence of $\widehat{R}$-module homomorphisms,

$$
0 \rightarrow \operatorname{Hom}_{R}(\widehat{R}, K) \rightarrow \operatorname{Hom}_{R}(\widehat{R}, E) \rightarrow \operatorname{Ext}_{R}^{1}(\widehat{R}, R) \rightarrow 0 .
$$

Since $K$ and $E$ are injective over $R$, the modules $\operatorname{Hom}_{R}(\widehat{R}, K)$ and $\operatorname{Hom}_{R}(\widehat{R}, E)$ are injective over $\widehat{R}$. Because $\operatorname{Hom}_{R}(\widehat{R}, K)$ is injective over $\widehat{R}$, the sequence (4.2.2) splits. As $\operatorname{Hom}_{R}(\widehat{R}, E)$ is injective over $\widehat{R}$, it follows that $\operatorname{Ext}_{R}^{1}(\widehat{R}, R)$ is injective over $\widehat{R}$. So, there is an $\widehat{R}$-module isomorphism

$$
\operatorname{Ext}_{R}^{1}(\widehat{R}, R) \cong E^{(D)} \oplus \widetilde{K}^{(C)}
$$


for cardinals $C$ and $D$ where $D=\operatorname{dim}_{k}\left(\operatorname{Hom}_{\widehat{R}}\left(k, \operatorname{Ext}_{R}^{1}(\widehat{R}, R)\right)\right)$. Since the sequence (4.2.2) splits, we have the third step in the next sequence:

$$
\begin{aligned}
k & \cong \operatorname{Hom}_{R}(k, E) \\
& \cong \operatorname{Hom}_{\widehat{R}}\left(k, \operatorname{Hom}_{R}(\widehat{R}, E)\right) \\
& \cong \operatorname{Hom}_{\widehat{R}}\left(k, \operatorname{Hom}_{R}(\widehat{R}, K)\right) \oplus \operatorname{Hom}_{\widehat{R}}\left(k, \operatorname{Ext}_{R}^{1}(\widehat{R}, R)\right) \\
& \cong \operatorname{Hom}_{R}(k, K) \oplus \operatorname{Hom}_{\widehat{R}}\left(k, \operatorname{Ext}_{R}^{1}(\widehat{R}, R)\right) \\
& \cong \operatorname{Hom}_{\widehat{R}}\left(k, \operatorname{Ext}_{R}^{1}(\widehat{R}, R)\right) .
\end{aligned}
$$

The second and fourth steps follow by Hom-tensor adjointness, and the fifth step follows from the vanishing $\operatorname{Hom}_{R}(k, K)=0$ noted at the beginning of the proof. It follows that $D=1$, so the claim follows from the isomorphism (4.2.3).

Now we complete the proof of the proposition. Because of Claim 4 , we need only show that $C$ is uncountable. Consider the exact sequence

$$
0 \rightarrow R \rightarrow \widehat{R} \rightarrow \widehat{R} / R \rightarrow 0
$$

and part of the associated long exact sequence induced by $\operatorname{Ext}_{R}(\widehat{R},-)$ :

$$
\operatorname{Hom}_{R}(\widehat{R}, R) \rightarrow \operatorname{Hom}_{R}(\widehat{R}, \widehat{R}) \rightarrow \operatorname{Hom}_{R}(\widehat{R}, \widehat{R} / R) \rightarrow \operatorname{Ext}_{R}^{1}(\widehat{R}, R) \rightarrow \operatorname{Ext}_{R}^{1}(\widehat{R}, \widehat{R}) .
$$

Over $R$, this sequence has the following form by Claims 1 and 3 and Lemma 4.1 (c):

$$
0 \rightarrow \widehat{R} \rightarrow(\widetilde{K} / K)^{(B)} \rightarrow \operatorname{Ext}_{R}^{1}(\widehat{R}, R) \rightarrow 0 .
$$

Apply the functor $(-)_{X}$ to obtain the exact sequence of $K$-module homomorphisms,

$$
0 \rightarrow \widetilde{K} \rightarrow(\widetilde{K} / K)^{(B)} \rightarrow \operatorname{Ext}_{R}^{1}(\widehat{R}, R)_{X} \rightarrow 0,
$$

which therefore splits. Since $E_{X}=0$, it follows from Claim 4 that over $R$ we have

$$
\widetilde{K}^{(C)} \cong \operatorname{Ext}_{R}^{1}(\widehat{R}, R)_{X} \cong(\widetilde{K} / K)^{(B)} / \widetilde{K} \cong\left(K^{(A)}\right)^{(B)} / K^{(A)} \cong K^{(B)} / K^{(A)} \cong K^{(B)} \text {. }
$$

The last two steps in this sequence follow from the fact that $A$ and $B$ are infinite cardinals such that $B>A$.

Suppose that $C$ were countable. It would then follow that $C \leqslant A$, so we have

$$
K^{(B)} \cong \widetilde{K}^{(C)} \cong\left(K^{(A)}\right)^{(C)} \cong K^{(A)} .
$$

It follows that $B=A$, contradicting the fact that $B>A$. It follows that $C$ is uncountable, as desired.

Remark 4.3. Nagata [11, (E3.3)] shows that the assumption $[\widetilde{K}: K]=\infty$ in Proposition 4.2 is not automatically satisfied. On the other hand, the next result shows that the condition $[\widetilde{K}: K]=\infty$ is satisfied by the rings $\mathbb{Z}_{p \mathbb{Z}}$ and $R=k[X]_{(X)}$.

Proposition 4.4. Assume that $R$ is a discrete valuation ring with $\mathfrak{m}=X R$ and such that $|R|=|k|$. For the quotient fields $K=Q(R)$ and $\widetilde{K}=Q(\widehat{R})$, we have $[\widetilde{K}: K]=\infty$.

Proof. We claim that $|\widehat{R}|>|R|$. To show this, let $\left\{a_{t}\right\}_{t \in k} \subseteq R$ be a set of representatives of the elements of $k$. Then every element of $\widehat{R}$ has a unique representation $\sum_{i=0}^{\infty} a_{t_{i}} X^{i}$. It follows that $|\widehat{R}|=|k|^{\aleph_{0}}>|k|=|R|$, as claimed.

Suppose now that $[\widetilde{K}: K]=A<\infty$. The fact that $K$ is infinite implies that

$$
|K|=A|K|=|\widetilde{K}|=|\widehat{R}|>|R|=|K|,
$$

a contradiction. 
The proof of Proposition 4.2 translates directly to give the following.

Proposition 4.5. Assume that $R$ is a discrete valuation ring that is not complete, with $\mathfrak{m}=X R$. Set $E=E_{R}(k)=E_{\widehat{R}}(k)$, and consider the quotient fields $K=Q(R)$ and $\widetilde{K}=Q(\widehat{R})$. If $[\widetilde{K}: K]=A<\infty$, then there are $\widehat{R}$-module isomorphisms

$$
\operatorname{Ext}_{R}^{i}(\widehat{R}, R) \cong \begin{cases}0 & \text { if } i \neq 1 \\ E \oplus \widetilde{K}^{A-2} & \text { if } i=1 .\end{cases}
$$

Remark 4.6. It is worth noting that in the notation of Proposition 4.5 we cannot have $A=1$. Indeed, if $A=1$, then we have $\widetilde{K}=K$, and the proof of Proposition 4.5 shows that $\widehat{R} / R \cong \widetilde{K} / K=0$, contradicting the assumption that $R$ is not complete.

On the other hand, Nagata [11, (E3.3)] shows how to build a ring $R$ such that $A=2$, which then has $\operatorname{Ext}_{R}^{i}(\widehat{R}, R) \cong E$ by Proposition 4.5 .

\section{ACKNOWLEDGMEnTS}

The authors are grateful to a-fortiori@mathoverflow .net, Phil Griffith, Bruce Olberding, and Irena Swanson for helpful discussions about this material. They also thank the anonymous referee for valuable comments and suggestions.

\section{REFERENCES}

[1] Luchezar L. Avramov, Ragnar-Olaf Buchweitz, and Liana M. Şega, Extensions of a dualizing complex by its ring: commutative versions of a conjecture of Tachikawa, J. Pure Appl. Algebra 201 (2005), no. 1-3, 218-239, DOI 10.1016/j.jpaa.2004.12.029. MR2158756(2006e:13012)

[2] Ragnar-Olaf Buchweitz and Hubert Flenner, Power series rings and projectivity, Manuscripta Math. 119 (2006), no. 1, 107-114, DOI 10.1007/s00229-005-0608-8. MR2194381 (2007a:13025)

[3] Lars Winther Christensen, Gorenstein dimensions, Lecture Notes in Mathematics, vol. 1747, Springer-Verlag, Berlin, 2000. MR 1799866 (2002e:13032)

[4] Lars Winther Christensen and Sean Sather-Wagstaff, Transfer of Gorenstein dimensions along ring homomorphisms, J. Pure Appl. Algebra 214 (2010), no. 6, 982-989, DOI 10.1016/j.jpaa.2009.09.007. MR2580673 (2011b:13047)

[5] H.-B. Foxby, Hyperhomological algebra and commutative rings, lecture notes.

[6] Hans-Bjørn Foxby and Srikanth Iyengar, Depth and amplitude for unbounded complexes, Commutative algebra (Grenoble/Lyon, 2001), Contemp. Math., vol. 331, Amer. Math. Soc., Providence, RI, 2003, pp. 119-137, DOI 10.1090/conm/331/05906. MR2013162 (2004k:13028)

[7] Anders J. Frankild and Sean Sather-Wagstaff, Detecting completeness from Ext-vanishing, Proc. Amer. Math. Soc. 136 (2008), no. 7, 2303-2312, DOI 10.1090/S0002-9939-08-09199-5. MR2390496 (2009c:13016)

[8] Anders J. Frankild, Sean Sather-Wagstaff, and Roger Wiegand, Ascent of module structures, vanishing of Ext, and extended modules, Special volume in honor of Melvin Hochster, Michigan Math. J. 57 (2008), 321-337, DOI 10.1307/mmj/1220879412. MR2492456(2010k:13025)

[9] Birger Iversen, Amplitude inequalities for complexes, Ann. Sci. École Norm. Sup. (4) 10 (1977), no. 4, 547-558. MR0568903 (58 \#27966)

[10] Hideyuki Matsumura, Commutative ring theory, 2nd ed., Cambridge Studies in Advanced Mathematics, vol. 8, Cambridge University Press, Cambridge, 1989. Translated from the Japanese by M. Reid. MR1011461 (90i:13001)

[11] Masayoshi Nagata, Local rings, Interscience Tracts in Pure and Applied Mathematics, No. 13, Interscience Publishers, a division of John Wiley \& Sons, New York-London, 1962. MR0155856 (27 \#5790) 
Department of Mathematics, North Dakota State University, Department \#2750, P.O. Box 6050, FArgo, North DAKota 58108-6050

E-mail address: benjamin.j.anderson@ndsu.edu

$U R L:$ http://www.ndsu.edu/pubweb/ ^benjaand/

Current address: University of Wisconsin-La Crosse, 1725 State Street, La Crosse, Wisconsin 54601

E-mail address: banderson@uwlax.edu

Department of Mathematics, North Dakota State University, Department \#2750, P.O. Box 6050, FArgo, North Dakota 58108-6050

E-mail address: sean.sather-wagstaff@ndsu.edu

URL: http://www.ndsu.edu/pubweb/〜 ssatherw/ 